\title{
Electromagnetic Design of a Magnetically- Coupled Spatial Power Combiner
}

Bulcha, B., Cataldo, G, Stevenson, T.R., U-Yen, K., Moseley, S.H., Wollack, E.J.

Presented by: Berhanu Bulcha (PhD)

NASA Goddard Space Flight Center

07/15/2017 


\section{Introduction}

- There is a high need to understand the early universe.

- Energy released from the early universe has been redshifted into the far-infrared.

- Stars and planets in the early stages of formation and the powerful cores of active galaxies, emit most strongly in the infrared.

- The ability to fully explore this rich spectral region has been limited by the size, mass, power constraints and limitations in sensitivity.

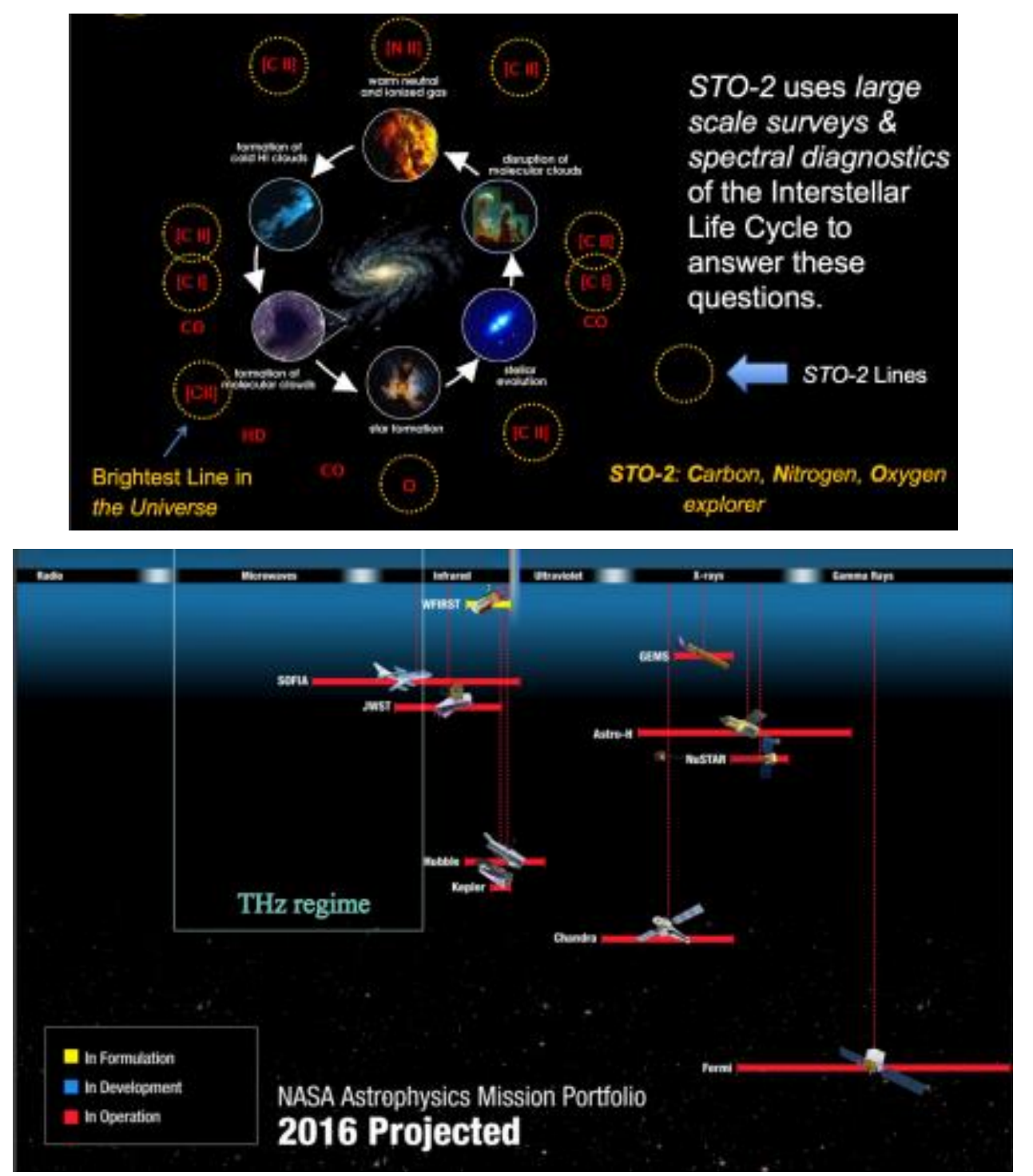




\section{A Far-IR Spectrometer: Micro-Spec}

- Compact, ultra-low-noise, direct-detection spectrometers are compiling means to achieve these science goals.

- A high resolution spectrometer know as Micro-Spec (u-spec), operating in the $300-600 \mathrm{GHz}$ range is under development.

- $\mu$-spec is fabricated on 100-mm-diameter mono-crystalline silicon chip.

- Radiation is collected using a broadband slot antenna followed by power splitter and delay networks.

- The transmitting antenna radiating the signal through the $2 \mathrm{D}$ multimode region and the beam is combined at the receiving antenna as a function of frequency.

- A broadband absorber in the multimode region wall is used to reduce and control scattering.

- The signal will go through an order-sorting

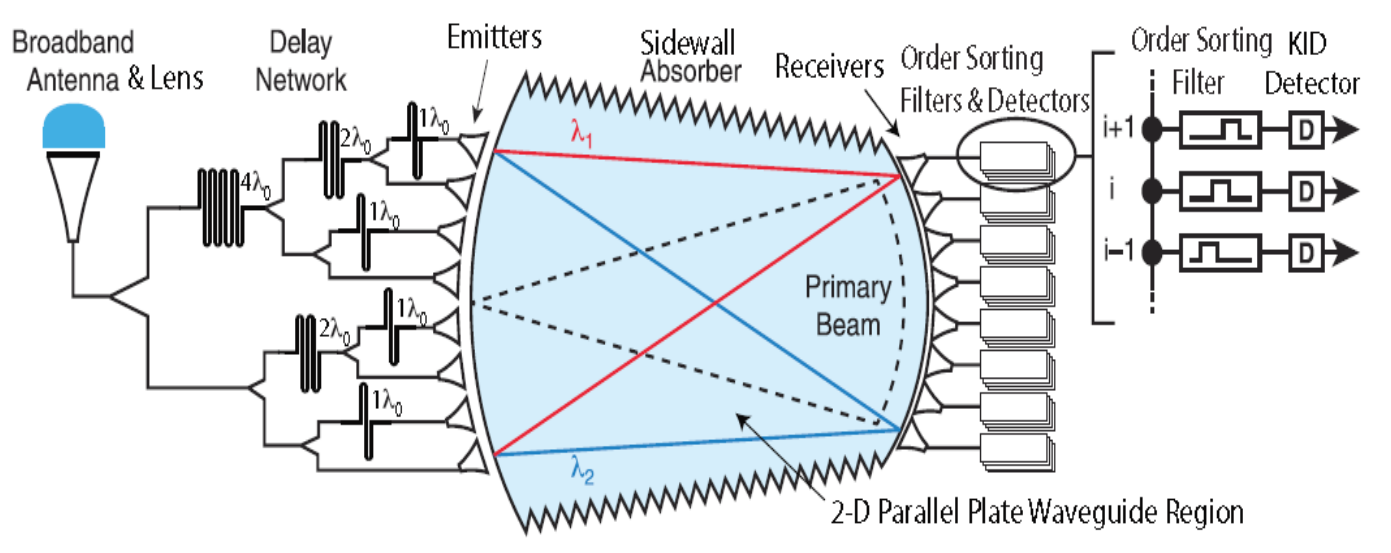
filter and will be detected using an array of MKIDs. 


\section{Transmitter and Receiver}

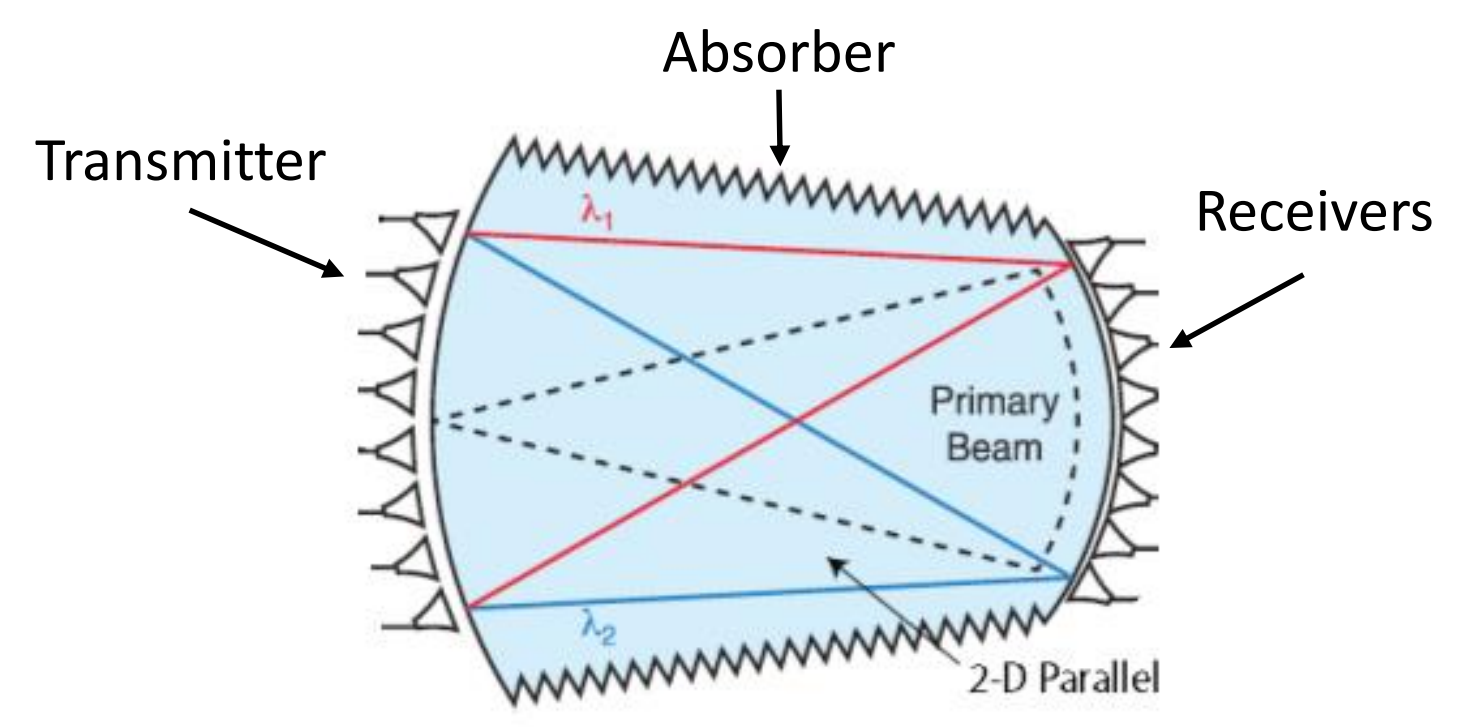

- The new $\mathrm{R}=512$ spectrometer will use the magnetically coupled feed antenna array and a broadband absorber.

- The magnetically coupled feed provides

- High coupling efficiency between transmit and receive arrays in the power combiner.

- Wide spectral bandwidth (1.7:1).

- low insertion loss (reflective mismatch). 


\section{Transmitter and Receiver cont...}

- Due to the diffractive losses In the aperture coupled configuration, the efficiency rapidly drops when twice the aperture size exceeds the radiation wavelength.

- These physical limitations are the challenge that needs to be addressed in the multi-mode power combiner to achieve near unity coupling over a broadband.

- The magnetically coupled antenna feed is a two dimensional array power combiner that would address these challenges by reducing the spaceing.

- Design:

- Two-dimensional parallel plate waveguide

- Operates 300-600 GHz

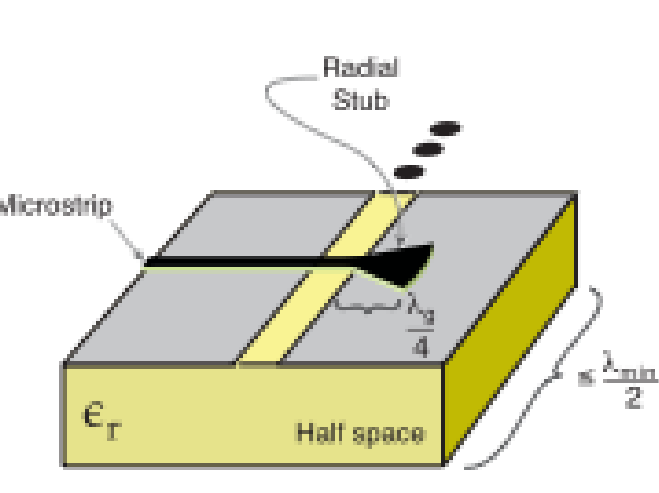

Longslot Coupled Element

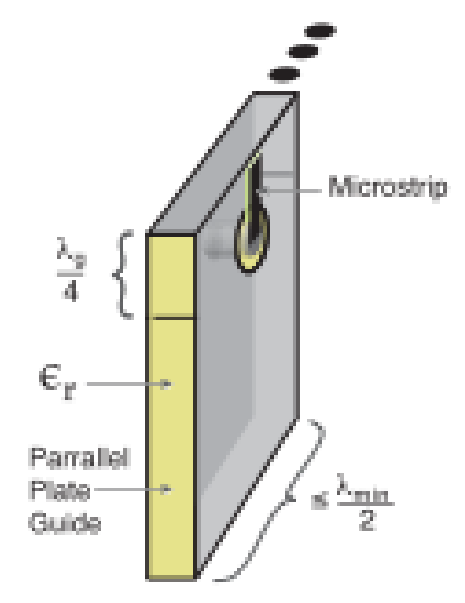

Magnetically Coupled Element

- Realized through the use of long slot antenna

- Each magnetically coupled feed is achieved by utilizing a via between microstrip line and the ground plane in the multimode region. 


\section{Magnetically Coupled Antenna Design}

- A single element and array of the structure are designed using a High Frequency Structural Software (HFSS).

- $420 \mu \mathrm{m}$ Silicon handling Substrate, $\mathrm{Nb}=500 \mathrm{~nm}$ thick conductor with Tc 9.2K, RRR > = 5; and Kinetic Inductance, $L k=0.084 \mathrm{pH} /$ square.

- For the single element simulation, perfect $\mathrm{H}$ boundaries are used.

- Near-field beam pattern is computed.

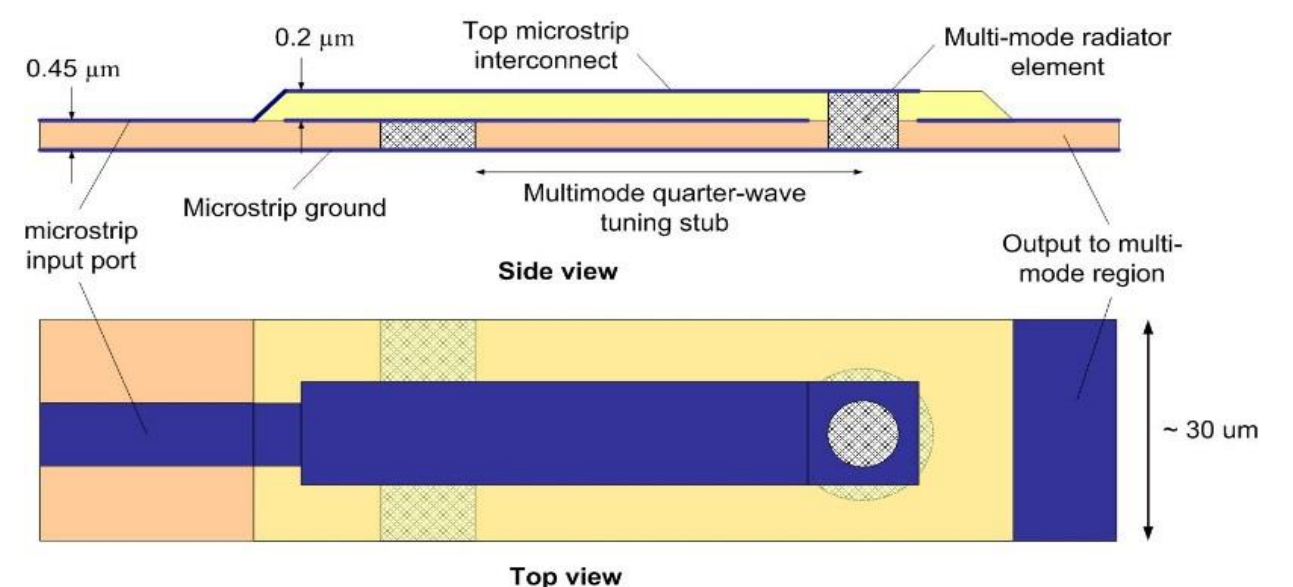

Top view

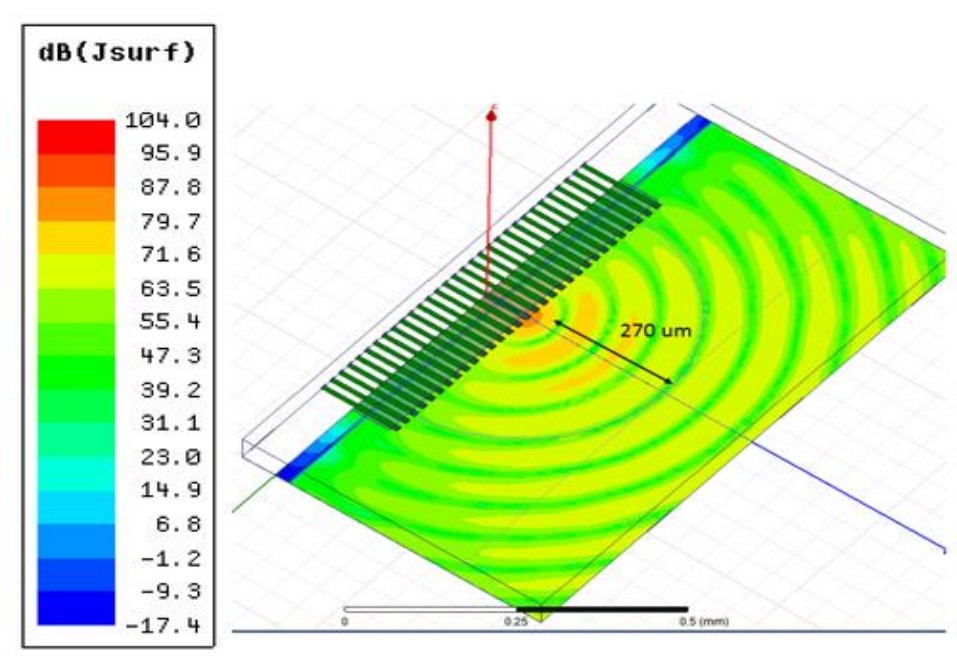

ए. Conductor filling $\square{\text { Top } \mathrm{SiO}_{2} \quad \square}_{\text {dielectric }}^{\text {Bottom Silcon }}$ - Superconductor 


\section{Return Loss Performance}

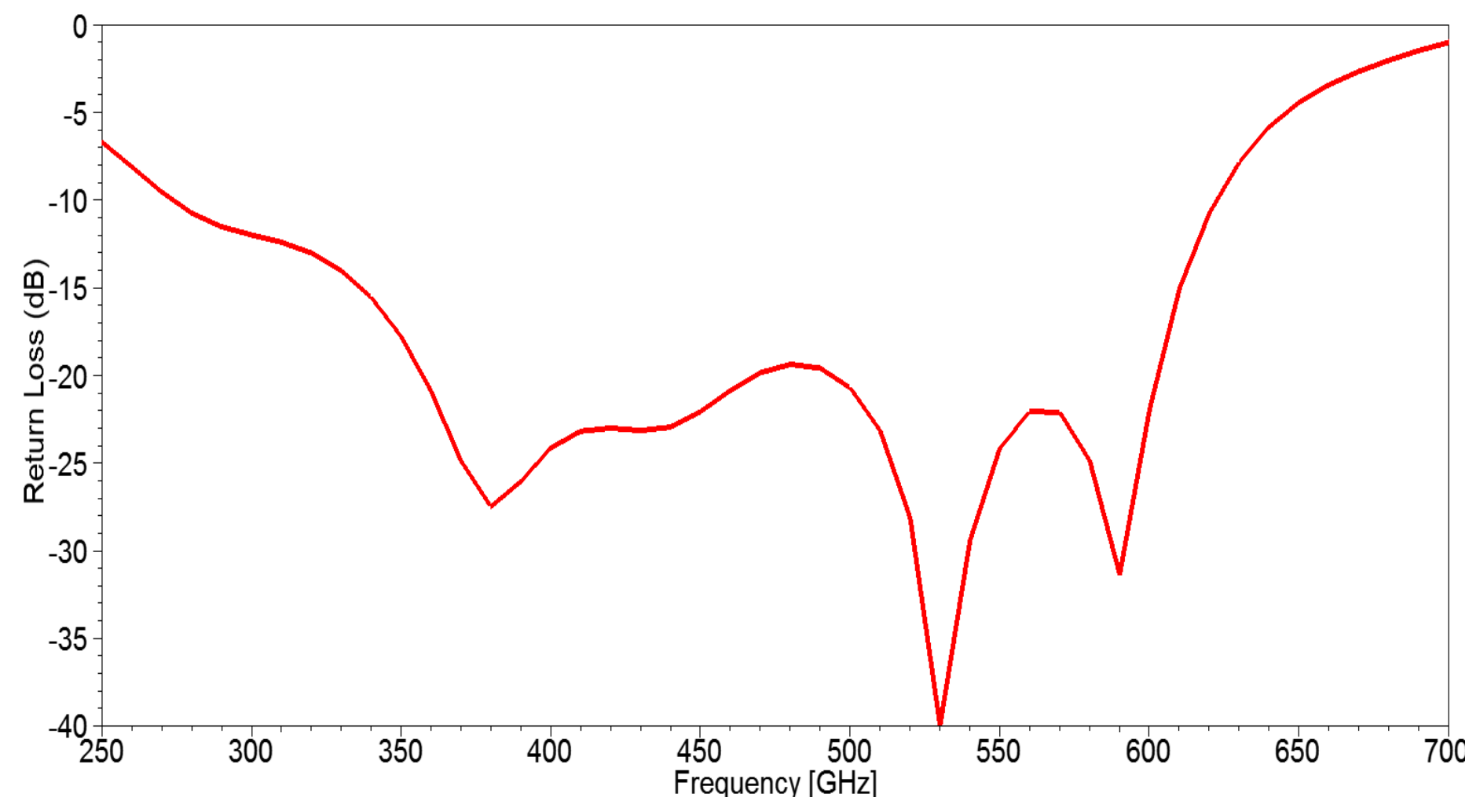

- Return loss better than $20 \mathrm{~dB}$

- Inter-feed isolation of $40 \mathrm{~dB}$ 


\section{Far-Field Beam}

- The near field simulated data from HFSS is used to create the far-field beam pattern using MATLAB assuming a cylindrical symmetry.

$$
\begin{aligned}
& \rho\left(x_{0}, y_{0}\right)=\rho_{\text {out }}\left(x_{0}, y_{0}\right)+\rho_{\text {in }}\left(x_{0}, y_{0}\right) \\
& \rho_{\text {out }}\left(x_{0}, y_{0}\right) \approx \sqrt{\frac{k}{8 \pi R_{0}}} e^{-j\left(k k \phi_{p}+3 \pi / 4\right)} \int_{-\infty}^{\infty} e^{j k \sin \theta}\left[\rho(x, 0) \cos \theta+\frac{1}{v} K_{y}(x, 0)\right] d x \\
& \rho_{\text {in }}\left(x_{0}, y_{0}\right) \approx \sqrt{\frac{k}{8 \pi R_{0}}} e^{-j\left(k R_{0}+3 z \pi / 4\right)} \int_{-\infty}^{\infty} e^{j / x \sin \theta}\left[\rho(x, 0) \cos \theta-\frac{1}{v} K_{y}(x, 0)\right] d x
\end{aligned}
$$

- A single element is activated with $1 \mathrm{~W}$ input power.

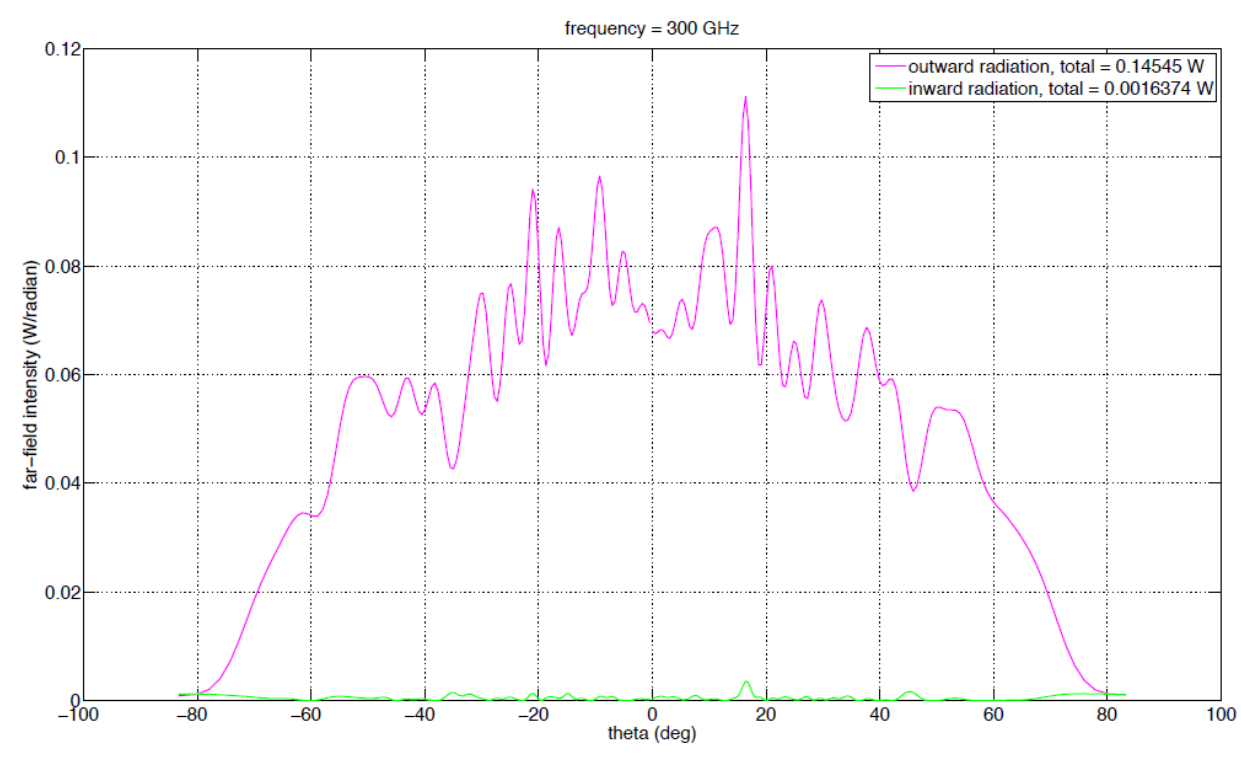

- Activating 4 elements will produce $86 \%$ efficiency at $300 \mathrm{GHz}$. 


\section{Broadband Absorber}

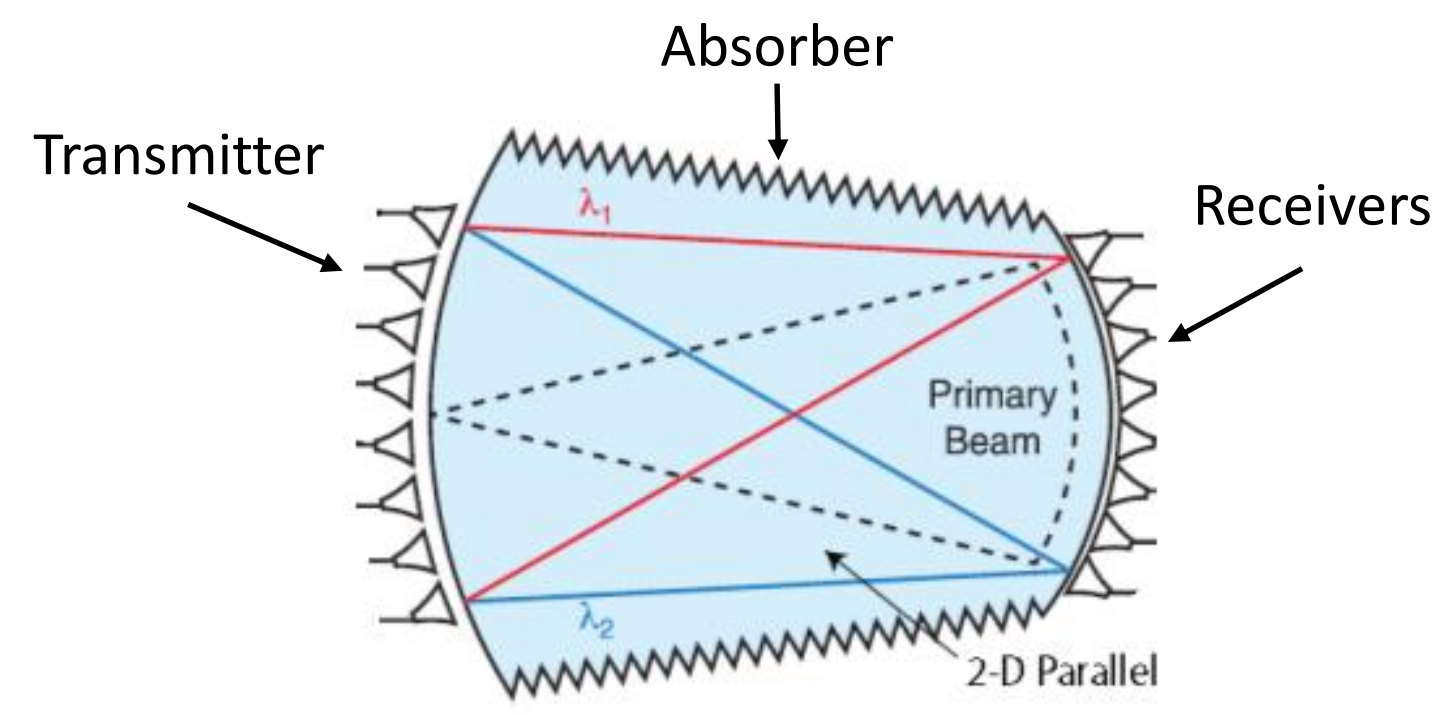

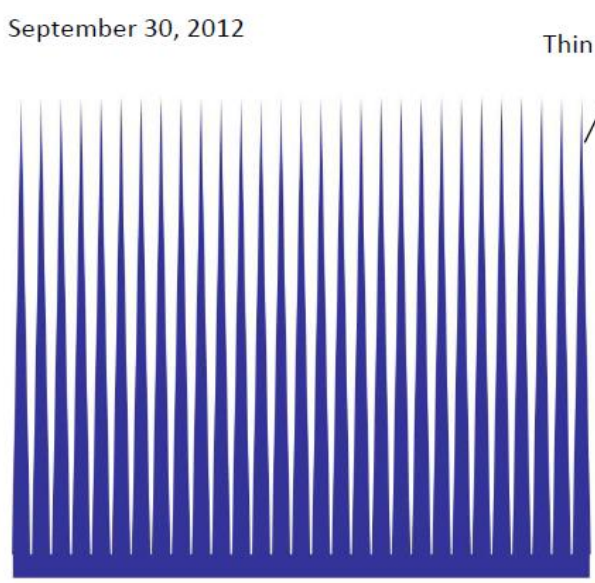

Triangular resistor tiling absorber
Circular resistor tiling absorber

(b)

- Propagation in a waveguide requires proper termination of signals to prevent reflections from interfaces.

- Planar termination designs such as triangular resistor films for 2-D can achieve a broad absorptive, however, it exhibit poor performance when signal is incident at modest angle.

- Our objective is to make a wide angle and broadband adiabatic signal termination. 


\section{Broadband Absorber}

-When the circular resistors are placed evenly and increased in size along the plane wave direction, the structure behaves as a dissipative impedance transformer.

- The size of the circle must be less than half the effective wavelength of the plane wave in dielectric medium at the maximum operating frequency to prevent signal reflection and higher mode generation.

- The low-end of its operating frequency band is set by the length of the array of resistors.

- The spacing between each unit cell sets the upper operating band-edge.

- Sheet resistor of $20 \mathrm{Ohm} / \mathrm{square}$.

- Minimum return loss (<-20dB).

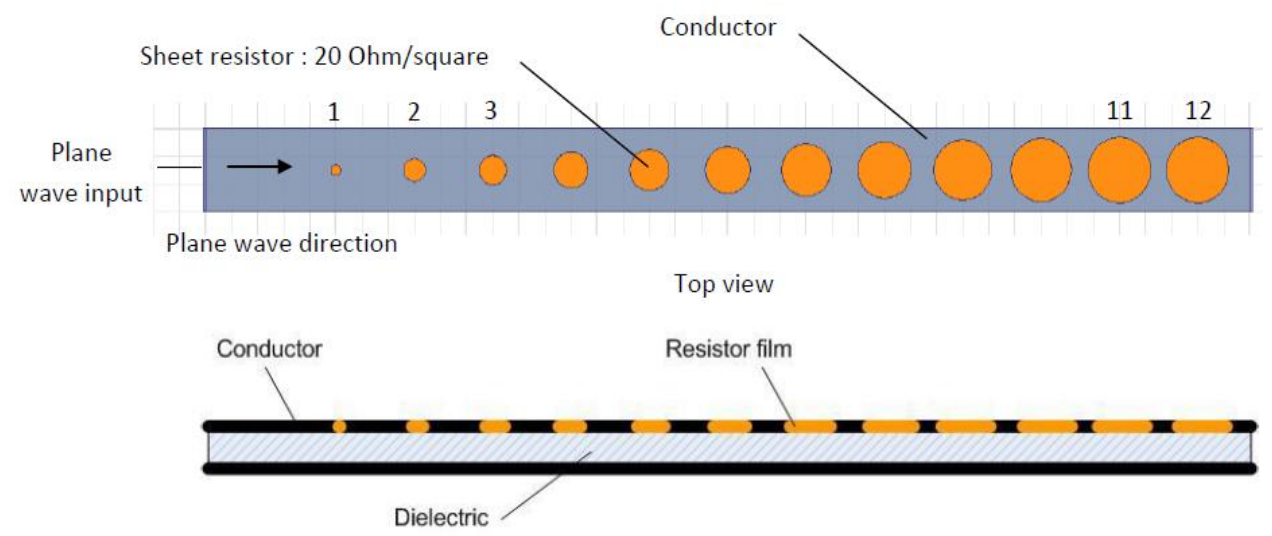




\section{Return Loss vs. Number of Sections}

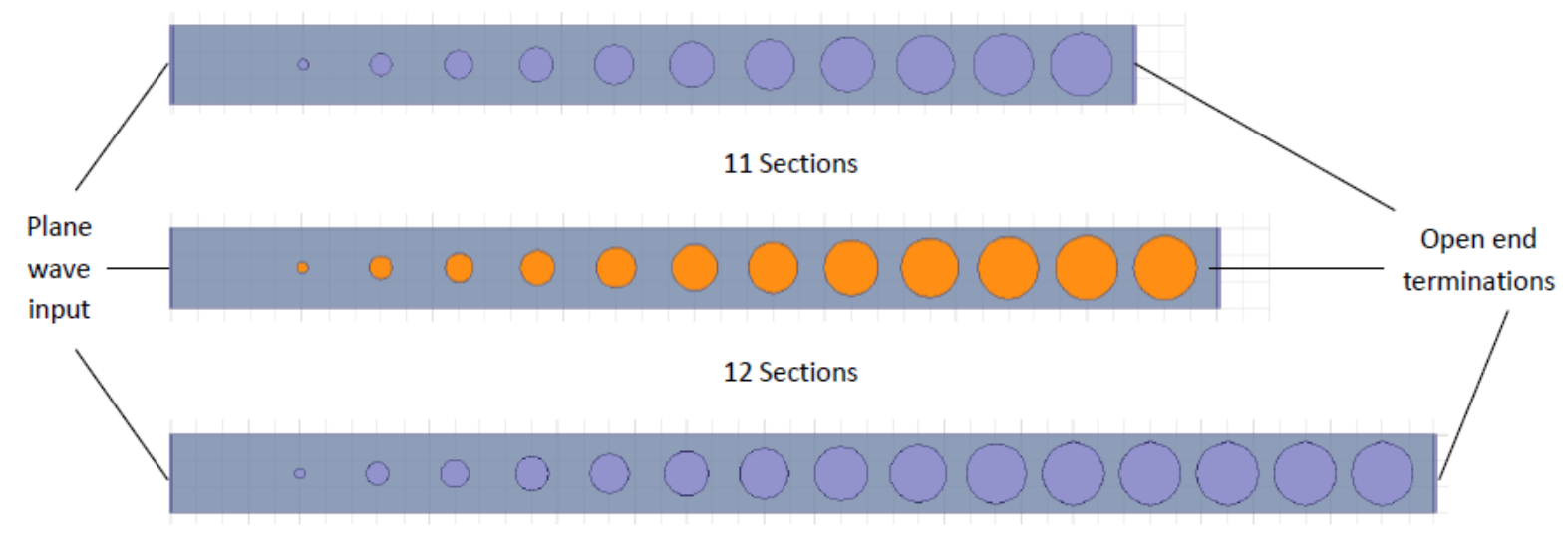

15 Sections

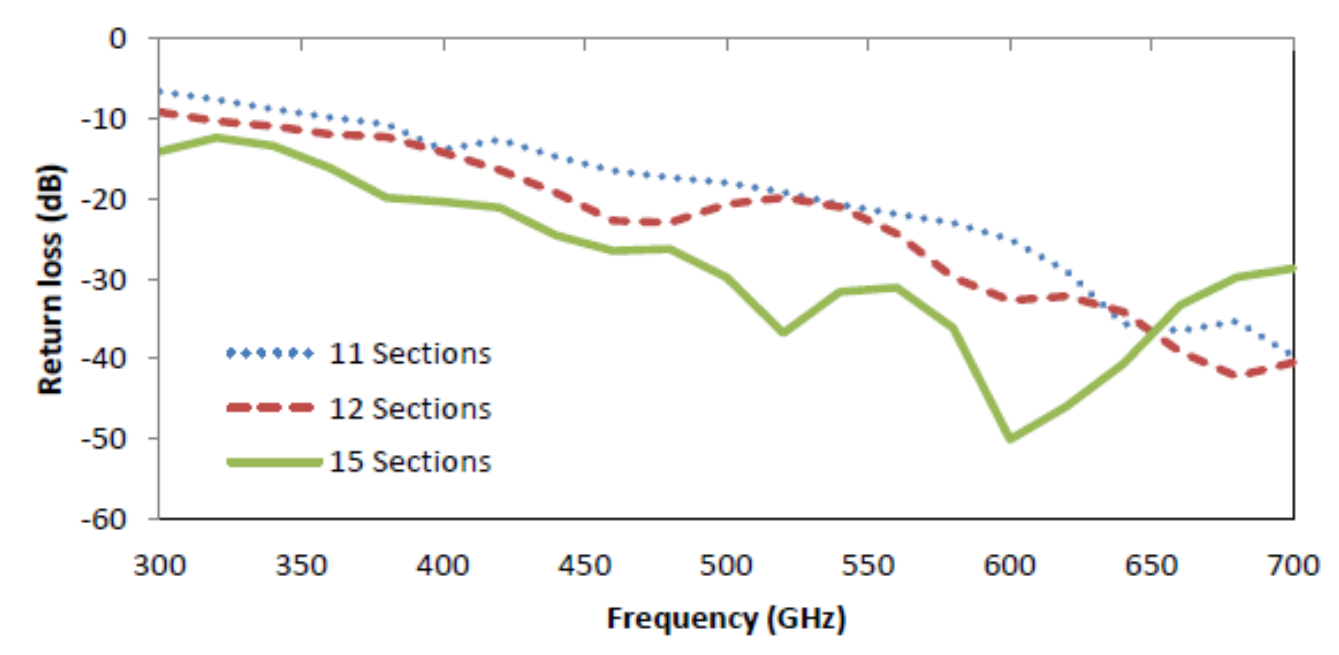

- Sheet resistor of $20 \mathrm{Ohm} / \mathrm{square}$.

- Minimum return loss (<-10dB)

- Designs with 11 to 15 resistor sections were explored to achieve the desired attenuation. 


\section{Absorption at Different Angles}

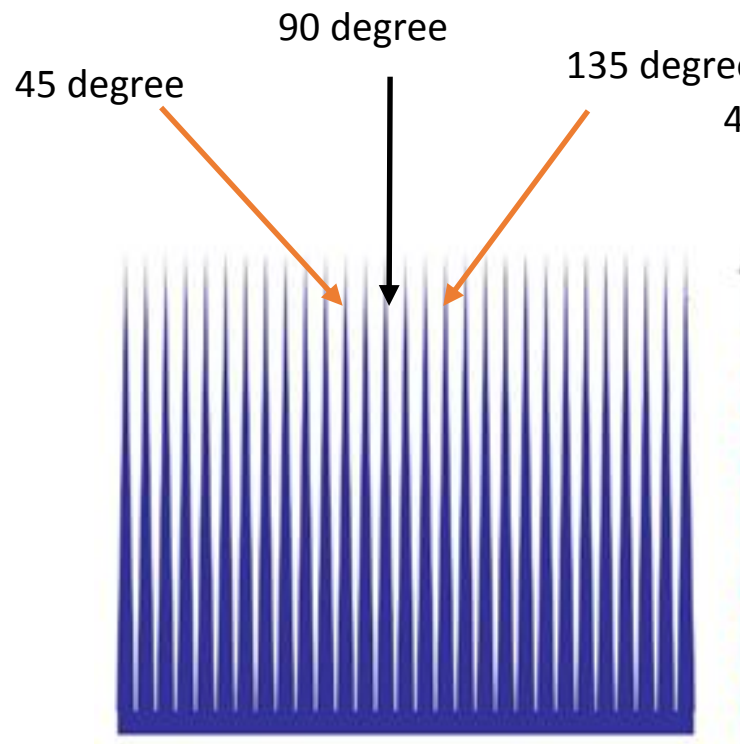

Triangular Resistor Tiling absorber
90 degree 45 degree r
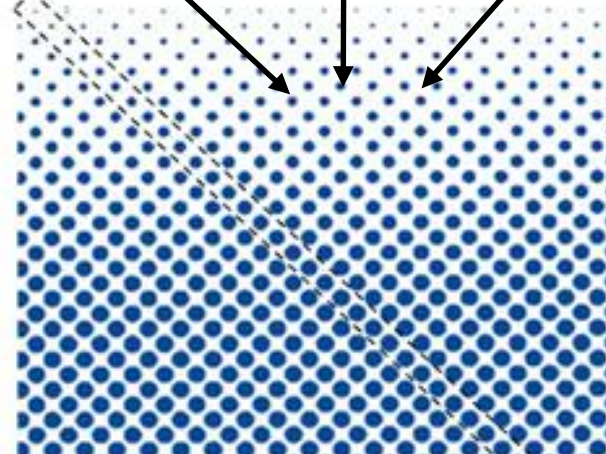

Circular Resistor Tiling absorb́er

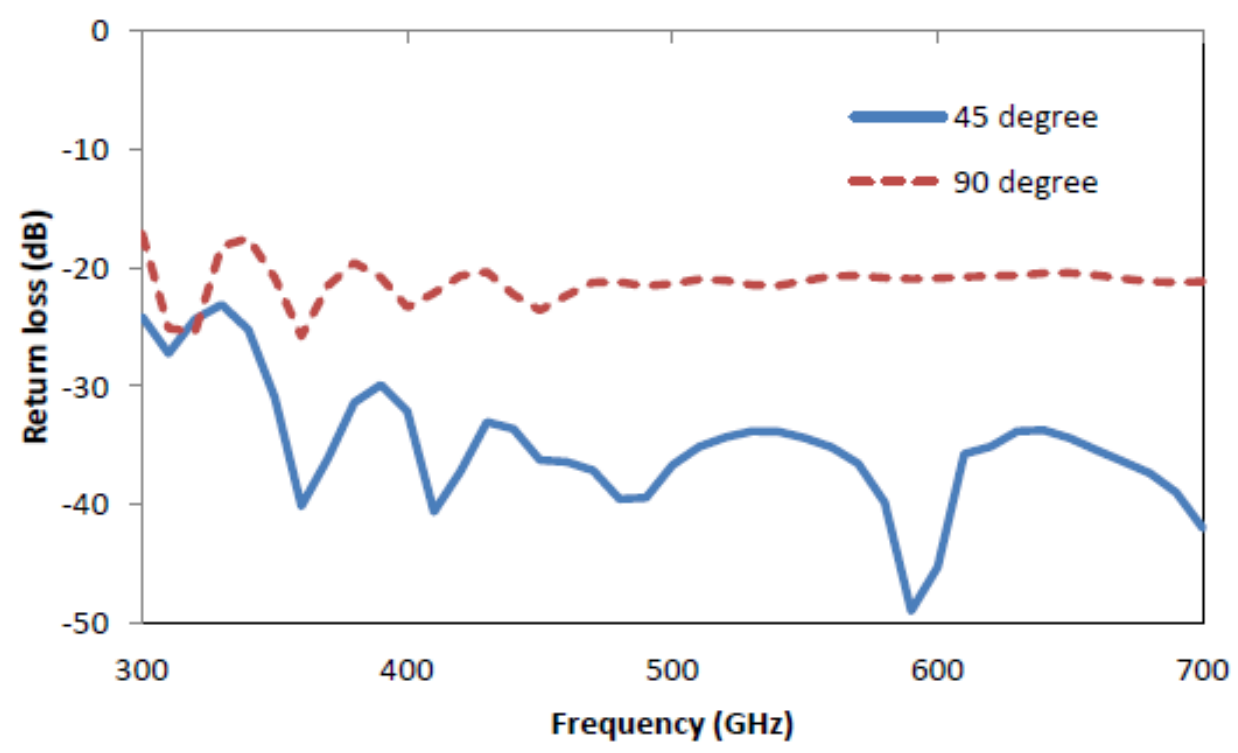

135 degree

- Absorber width of $400 \mu \mathrm{m}$

- 300-600 GHz

- Simulated frequency response of the absorber at 45 and 90 degrees using 21 sections of resistor circles

- The design can effectively absorb more than $99 \%$ of plane wave signal in the operating band.

- The absorber structure was optimized for minimal reflectance for a given wafer footprint. The metamaterial design concept is more general, and for example it would allow optimization for angle independent absorption. 


\section{Conclusion}

-We are developing a high resolution, compact, ultra-low-noise, directdetection spectrometers operating in the $300-600 \mathrm{GHz}$ range.

- The new $R=512$ spectrometer will use the magnetically coupled feed antenna array and a broadband absorber.

- The magnetically coupled feed antenna near-field is simulated and the farfield is computed.

- A wide angle and broadband signal absorber is designed. 